
\section{$C_{\text {cotuse }}$}

\begin{abstract}
- Additional material is published online only. To view please visit the journal online (http://dx.doi.org/10.1136/ thoraxjnl-2017-211243)
\end{abstract}

${ }^{1}$ Monash School of Medicine, Monash University, Melbourne, Australia

${ }^{2}$ Department of Respiratory and Critical Care Medicine, Singapore General Hospital, Singapore, Singapore

\section{Correspondence to} Dr Anne Ann Ling Hsu Department of Respiratory and Critical Care Medicine Singapore General Hospital, Singapore 169856, Singapore; anne.hsu.a.|@sgh.com.sg

Received 2 November 2017 Revised 29 December 2017 Accepted 5 January 2018 Published Online First 20 January 2018

\title{
Dieulafoy's disease with mediastinal arteriovenous malformation
}

\author{
Andrea Zhi Xin Foo, ${ }^{1}$ Anne Ann Ling Hsu ${ }^{2}$
}

A 42-year-old, non-smoker man was hospitalised for newly diagnosed acute myeloid leukaemia and had an episode of haemoptysis of $200 \mathrm{~mL}$ fresh blood, complicated by transient hypotension. Patient was afebrile and his blood results were: haemoglobin $10.0 \mathrm{~g} / \mathrm{dL}$ decreasing to $8.5 \mathrm{~g} / \mathrm{dL}$ within 1 day of haemoptysis, total white cell counts $23 \times 10^{9} / \mathrm{L}$, platelet counts $30 \times 10^{9} / \mathrm{L}$, normal renal panel and coagulation profile. CT scan reported extensive tortuous mediastinal vessels (figure 1A). Flexible bronchoscopy under platelet transfusion revealed a small lesion in the right upper lobe (RUL) bronchus (figure 2). Upon contact of the bronchoscope tip with the lesion, brisk bleeding occurred (online supplementary video) which ceased with ice-cold saline wash. A diagnosis of Dieulafoy's disease associated with mediastinal arteriovenous malformation (AVM) was made. CT angiogram followed by embolisation of major vessels feeding into RUL (figures 1B and 3) was performed. Embolisation was successful with the patient experiencing no further haemoptysis as he underwent intensive therapy for leukaemia in the following 1 year.

Dieulafoy's disease is a vascular anomaly distinguished by a tortuous, dysplastic artery in the submucosa. Although usually occurring in the gastrointestinal tract, cases of bronchial Dieulafoy's disease have been reported. ${ }^{1}$ Mediastinal AVM consists of multiple direct communications between arterial and venous branches, predominately in the posterior mediastinum. ${ }^{2}$ There is a scarcity of data
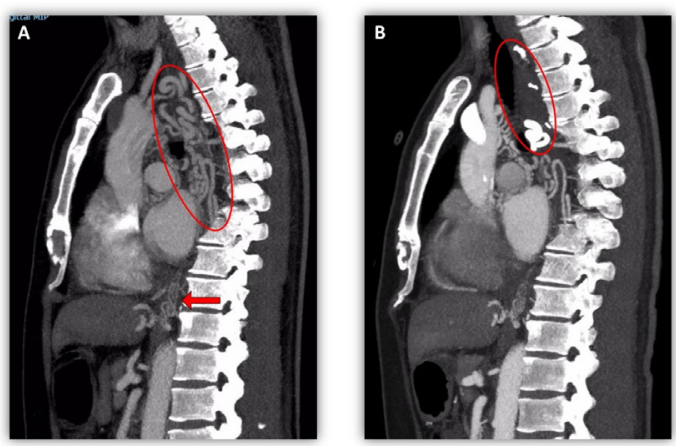

Figure 1 (A) Pre-embolisation CT scan without contrast revealed numerous dilated tortuous vessels predominantly in the posterior mediastinum (circle), suggestive of arteriovenous malformation (AVM). There were also serpiginous dilated arteries extending inferiorly along the lower paraoesophageal region (arrow). (B) Post-embolisation CT scan without contrast showed selective embolisation with opaque glue of major vessels feeding into the right upper lobe which resulted in the obliteration of distal perfusion (circle).

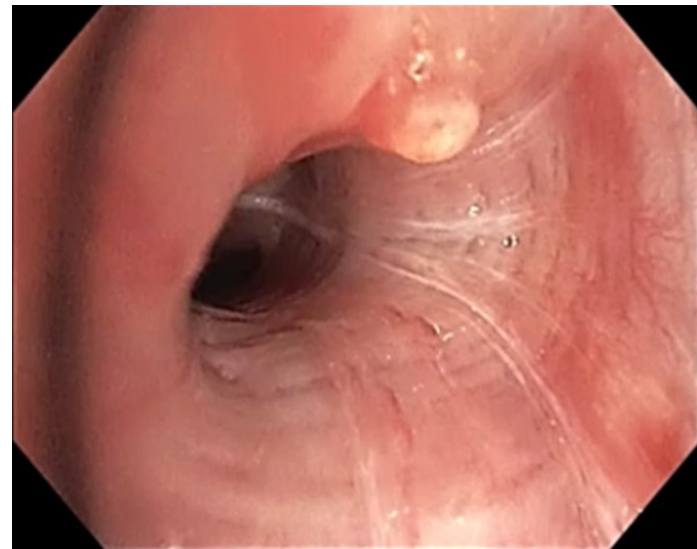

Figure 2 Sessile lesion with a whitish cap, a classic feature of Dieulafoy's disease, was detected in the right upper lobe anterior segmental bronchus during bronchoscopy.

reported in the literature on bronchial Dieulafoy's disease or mediastinal AVM; less than 20 cases of the latter have been discussed to date. To the best of our knowledge, reports of these dual rarities coexisting are hitherto absent. We present the first case report of life-threatening haemoptysis resulting from mediastinal AVM causing Dieulafoy's disease.

Of the few reports on bronchial Dieulafoy's disease causing haemorrhage, there are cases when active bleeding was triggered by iatrogenic injury, including bronchoscopic biopsy ${ }^{1}$ and saline wash. ${ }^{3}$ The effects of haematopoietic factor dysregulation, including
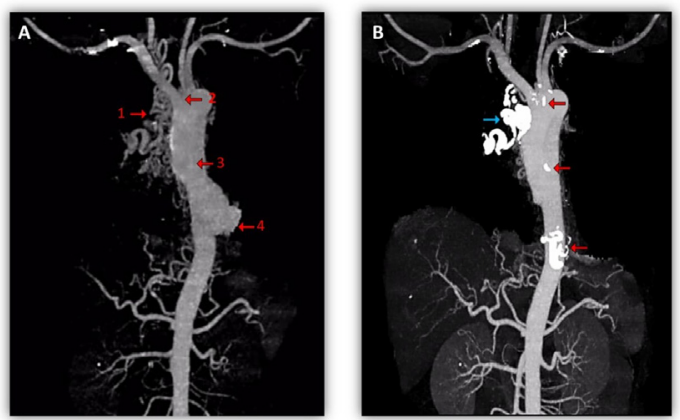

Figure 3 (A) Pre-embolisation angiogram identified four major mediastinal vessels with feeders from the right thyrocervical trunk (arrow 1), right internal mammary (2), bronchointercostal (3), inferior phrenic and branches from coeliac arteries (4) for embolisation. (B) Post-embolisation angiogram revealed successful embolisation with opaque glue of the four major vessels (arrows), including the artery feeding into the right upper lobe (blue arrow). 
qualitative and quantitative platelet defects, in acute leukaemia could have contributed to a heightened risk of bleeding in our patient. Both of these aspects were corrected by platelet transfusion during the procedure. Nevertheless, there should be earlier recognition before and greater caution should be taken during bronchoscopy. The nature of bleed and CT findings aroused suspicion of bronchial Dieulafoy's disease associated with AVM in this patient.

Selective angioembolisation should be considered as the firstline management for each of these two anomalies due to its minimal invasiveness, ${ }^{1-3}$ with angiography done prior to determine locality of the abnormal vessels. ${ }^{1}$ Surveillance of embolised vessels thereafter is recommended due to possible revascularisation and neovascularisation leading to failure of initially successful embolisation. If haemoptysis reoccurs, recurrent embolotherapy $^{3}$ and surgery should be considered. ${ }^{1}$

Contributors The article was conceived by AZXF and AALH. AALH provided materials for the article while AZXF was responsible for the draft preparation and revision. Both authors were involved in reviewing each draft and approving the final manuscript. AALH is the guarantor of the study.

Funding This research received no specific grant from any funding agency in the public, commercial or not-for-profit sectors.

Competing interests None declared.

Patient consent Next of kin consent obtained.

Provenance and peer review Not commissioned; externally peer reviewed.

(c) Article author(s) (or their employer(s) unless otherwise stated in the text of the article) 2018. All rights reserved. No commercial use is permitted unless otherwise expressly granted.

\section{REFERENCES}

1 Fang Y, Wu Q, Wang B. Dieulafoy's disease of the bronchus: report of a case and review of the literature. J Cardiothorac Surg 2014;9:191.

2 Tennyson C, Routledge T, Chambers A, et al. Arteriovenous malformation in the anterior mediastinum. Ann Thorac Surg 2010;90:e9-10.

3 Bhatia P, Hendy MS, Li-Kam-Wa E, et al. Recurrent embolotherapy in Dieulafoy's disease of the bronchus. Can Respir J 2003;10:331-3. 